\title{
Variation of Linear and Nonlinear Parameters in the Swim Strokes According to the Level of Expertise
}

\author{
Tiago M. Barbosa and Wan Xiu Goh \\ Nanyang Technological University \\ Jorge E. Morais \\ Polytechnic Institute of Bragança \\ Mário J. Costa \\ Polytechnic Institute of Guarda
}

\begin{abstract}
The aim was to examine the variation of linear and nonlinear proprieties of the behavior in participants with different levels of swimming expertise among the four swim strokes. Seventy-five swimmers were split into three groups (highly qualified experts, experts and nonexperts) and performed a maximal $25 \mathrm{~m}$ trial for each of the four competitive swim strokes. A speed-meter cable was attached to the swimmer's hip to measure hip speed; from which speed fluctuation (dv), approximate entropy (ApEn) and fractal dimension (D) variables were derived. Although simple main effects of expertise and swim stroke were obtained for $\mathrm{dv}$ and $\mathrm{D}$, no significant interaction of expertise and stroke were found except in ApEn. The ApEn and D were prone to decrease with increasing expertise. As a conclusion, swimming does exhibit nonlinear properties but its magnitude differs according to the swim stroke and level of expertise of the performer.
\end{abstract}

Keywords: swimming, nonlinear parameters, variability, predictability, complexity

To displace in water, a swimmer concurrently performs several limbs' motions that must be properly synchronized. These actions aim to increase the thrust (propulsive forces) and minimize the resistance (drag force) (Barbosa, Bragada, Reis, Marinho, Carvalho \& Silva, 2010a). Swim speed is the result of the interaction between these two external forces acting upon the subject within each stroke cycle and over different stroke cycles (Craig, Termin \& Pendergast, 2006). Therefore the assessment of the intracyclic and intercyclic variations of the swim speed is a

Barbosa and Goh are with the Nanyang Technological University, Singapore. Morais is with the Polytechnic Institute of Bragança, Bragança, Portugal. Costa is with the Polytechnic Institute of Guarda, Guarda, Portugal. Address author correspondence to Tiago M. Barbosa at tiago.barbosa@nie.edu.sg. 
topic of interest for several researchers (Seifert, Komar, Barbosa, Toussaint, Millet, \& Davids, 2014).

The intracyclic variation of the speed (dv) is monitored by the "speed fluctuation" (Barbosa, Bragada, Reis, Marinho, Carvalho \& Silva, 2010a). This is an index to quantify the changes of speed over a full stroke cycle. It expresses the ratio between the amount of variation or dispersion of the speed over an entire cycle and the mean speed. The dv falls under the field of "classic" linear kinematics. It has been reported by experimental research and numerical simulations that there is a trend for the dv to decrease with increasing speeds and it is different among the four swim strokes (Barbosa et al., 2013; Cohen, Cleary, Harrison, Mason \& Pease, 2014). This parameter is convenient for researchers and practitioners to have some insight on the forces acting upon the swimmer and the limbs' synchronization in a straightforward way (Craig, Termin \& Pendergast, 2006; Seifert, Komar, et al., 2014). However, others have concerns on the sensitivity of this parameter and suggested to alternatively assess the difference between maximal and minimal speed (Figueiredo, Kjendlie, Vilas-Boas, \& Fernandes, 2012), or the ratio of the difference between maximal and minimal speed with average speed (Psycharakis \& Sanders, 2009). Therefore, it seems that other parameters to learn about one's motor behavior should be explored.

"Nonlinear" dynamics presents a framework from which it is possible to investigate nonlinear parameters (Abarbanel, Rabinovich \& Sushchik, 1993). In a linear system, a small change in one input may have a small and easily quantifiable change in the output. On the other hand, nonlinear systems exhibit a very sensitive dependence on the inputs. Due to its sensitivity properties, linear measurements cannot identify small changes in the inputs that may lead to significant variations in the output. Two of the nonlinear parameters are the approximate entropy and the fractal dimension (Bravi, Longtin \& Seely, 2011). The approximate entropy (ApEn) is a nonlinear technique quantifying the temporal structure of the unpredictability in its fluctuations over a time-series dataset. Therefore, it can help in the understanding of the intercyclic variations over consecutive swim cycles. The fractal dimension (D) quantifies the complexity and irregularity of a time-series dataset. Thus, it can provide a different insight on the complexity of the intracyclic variations. The $\mathrm{dv}$ can be considered as an outcome of the swimming behavior. Behavioral parameters can exhibit nonlinear properties. Hence, dv may be the resultant of nonlinear properties in the behavior.

It is known that performance is strongly related to proportional changes in the inputs. The "marginal gains theory" in sports performance gained popularity a few years ago. It encompasses the assumption that small changes in the input (or the sum of several changes) may have a significant effect on the output. Hence, motor behavior in these settings clearly falls under nonlinear dynamics. To understand such complexity, irregularity and unpredictability of the motor behavior in these settings, fields such as mathematics, computer sciences and motor control may be quite useful. The more classical and more mainstream procedures may not be sensitive and insightful enough. Under this framework, it is advised that the selection of a couple of nonlinear measures concurrent to a linear parameter (e.g., for this case the $d v$ ) can have a more comprehensive understanding of the different motor behaviors (Neumeister, Cellucci, Rapp, Korn, \& Faber, 2004). Yet, only a few papers can be found in the literature reporting nonlinear parameters in sportsmen 
or sports techniques. With swimming as a challenging sport as explained earlier, it is ideal to assess nonlinear motor behaviors.

The ApEn has been selected for the analysis of youth and elderly gait (Arif, Ohtaki, Nagatomi \& Inooka, 2004), as well as young swimmers but only at Frontcrawl (Barbosa, Morais, Marques, Silva, Marinho \& Kee, 2015). It was reported that ApEn was higher for elderly than young subjects in walking at 100 steps/ minute and 120 steps/minute (Arif, Ohtaki, Nagatomi \& Inooka, 2004). In young swimmers, the ApEn decreased, becoming more predictble, over a season and this was coupled with a performance enhancement (Barbosa, Morais, Marques, Silva, Marinho \& Kee, 2015). Thus, both land-based and aquatic locomotion seem to involve a given degree of unpredictability. The unpredictability can be related to changes in the subject's biomechanics and motor control with an impact ultimately on his performance and motor behavior. These findings also suggest that there might be a relationship between the level of expertise and the ApEn. As far as swimming goes, this parameter can provide insight on the predictability of consecutive cycles. For instance, allowing us to understand the predictability of consecutive swim strokes over an entire lap. Hence, one can wonder if this predictability is higher or lower based on the level of expertise.

Fractal dimension has been reported for gait (Sekine, Tamura, Akay, Fujimoto, Togawa \& Fukui, 2002; Schiffman, Chelidze, Segala, \& Hasselquist, 2009), albeit not yet in swimming. Fractal dimension values tend to decrease over time from the beginning to end of a $120 \mathrm{~min}$ load carriage march (Schiffman, Chelidze, Segala, \& Hasselquist, 2009). Whereas, fractal dimension was significantly higher for elderly subjects than the young counterparts (Sekine, Tamura, Akay, Fujimoto, Togawa \& Fukui, 2002). The fractal dimension can enlighten us on the level of complexity of the motor behavior. However, caution should be exercised because very limited evidence is available on this topic yet. The findings on land-based locomotion point out that the level of expertise is related to the complexity of the motor behavior. However, it remains to be known if swimmers of different levels of expertise will likewise show different nonlinear behaviors or, if the magnitude of these parameters is different among the four swim strokes.

A better understanding of the behavioral complexity can provide valuable details in Nonlinear Pedagogy. The Nonlinear Pedagogy provides a practical framework for practitioners (Chow, Davids, Button, Shuttleworth, Renshaw \& Araújo, 2007). The principles of this pedagogical approach are based on the constraints-led approach (Davids, Hristovski, Araùjo, Balague Serre, Button, \& Passos, 2014). The latter encompasses key principles and features of the nonlinear dynamical systems. Therefore, the constraints-led approach provides the main theoretical framework, while the Nonlinear Pedagogy provides the practical framework with both embedded in the field of nonlinear dynamical systems.

The aim of this research was thus to examine the variation of linear and nonlinear behavior: (i) in subjects with different levels of swimming expertise and; (ii) among the four swim strokes. It was hypothesized that the behavior will be more predictable and less complex as the level of expertise increases and different among the four swim strokes. 


\section{Methods}

\section{Subjects}

A convenience sample of seventy-five swimmers was split into three groups of 25 subjects in accordance to their level of expertise (highly qualified experts, experts and nonexperts).

Subjects were assigned to one group based on their competitive level and therefore, swimming performance. Swimmers that compete on a regular basis at major international events (e.g., World Championships, continental and regional Games), hold national records and/or are national medallists were considered as "highly qualified experts" (21 males and 04 females, $15.73 \pm 1.52$ years old). Those competing on a regular basis at local competitions (e.g., national championships) but do not fall under the criteria reported earlier were assigned to the group of "expert" swimmers ( 11 males and 14 females, $15.74 \pm 3.55$ years old). "Non-expert" swimmers were subjects that practice the sport at a noncompetitive level on a regular basis (18 males and 07 females, $22.86 \pm 3.40$ years old).

The swimmers and when needed, coaches, parents or guardians, gave informed consent/assent for participation in this study. All procedures were in accordance with the Helsinki Declaration regarding human research. The University IRB also approved the research design.

\section{Protocol}

The swimmers performed a self-selected warm-up with a volume and intensity adjusted to their expertise and fitness level. Warm-up featured continuous swimming at low-moderate intensity, drills and sprints. Each swimmer undertook a set of maximal $4 \times 25 \mathrm{~m}$ swims (Front-crawl, Backstroke, Breaststroke and Butterfly strokes) after a push-off start, in a randomly assigned order (30min rest between trials). Participants performed each trial alone with no other swimmer in the lane or nearby lanes to minimize drafting, pacing effect or extra drag force acting upon the body. The swimmers were advised to start the swim stroke immediately after the push-off, hence minimizing gliding and not to perform underwater dolphin kicks.

\section{Data Collection}

A speedo-meter cord (Swim speedo-meter, Swimsportec, Hildesheim, Germany) was attached to the swimmer's hip (Barbosa et al., 2015). The speedo-meter was set on the forehead-wall of the swimming pool. A software interface in LabVIEW (v. 2015) was used to acquire $(f=50 \mathrm{~Hz})$, display and process speed-time data for each trial. Data were transferred from the speedo-meter to the software by a 12-bit acquisition card (USB-6008, National Instruments, Austin, Texas, USA). Then, data were exported to a signal processing software (AcqKnowledge v. 3.9.1, Biopac Systems, Santa Barbara, USA) and filtered with a $5 \mathrm{~Hz}$ cut-off low-pass fourth order Butterworth filter (beforehand it was plotted the residuals vs. cut-off frequency). The 
push-off start and the finish were discarded for the follow-up analysis and hence, not reflected in the parameters to be reported. To compute some of the nonlinear parameters, notably the ApEn, it is required to collect several consecutive cycles. For accuracy's sake, the algorithm required a minimum length in the dataset of at least 500 speed-time pairs. When benchmarked with other devices available to collect kinematic data (e.g., motion-capture systems or inertial measurement units), mechanical speedo-meters are the most convenient piece of equipment to collect such large datasets.

\section{Speed Fluctuation}

The intracyclic variation of the horizontal velocity of the hip $(d v)$ was computed as reported earlier (Barbosa et al., 2010a):

$$
d v=\frac{\sqrt{\frac{\sum_{i}\left(v_{i}-\bar{v}\right)^{2} \cdot F_{i}}{n}}}{\frac{\sum_{i} v_{i} \cdot F_{i}}{n}} \cdot 100
$$

Where $d v$ is the intracyclic variation of the horizontal velocity of the hip, $v$ is the mean swimming velocity, $v_{i}$ is the instant swimming velocity, $F_{i}$ is the acquisition frequency, and $n$ is the number of speed-time pairs. The $d v$ of three consecutive stroke cycles between the $11 \mathrm{thm}$ and $24 \mathrm{thm}$ from the starting wall were considered for further analysis.

\section{Approximate Entropy}

The intercyclic variation of the horizontal velocity of the hip was monitored by the ApEn and computed as (Pincus, 1991):

$$
\operatorname{ApEn}(N, m, r)=\ln \left[\frac{C_{m}(r)}{C_{m+1}(r)}\right]
$$

Where ApEn is the approximate entropy, $N$ is the data length $[N=700$ speed-time pairs, as suggested by Yentes, Hunt, Schmid, Kaipust, McGrath \& Stergiou (2013)], $m$ is the embedding dimension $(\mathrm{m}=2$, because two consecutive cycles contributing to two data points were considered for each mobile window), $r$ is the tolerance value or similarity criterion $[r=.1$, determined beforehand as the maximum $A p E n$ for a wide range of $r$ values between 0.01 and 0.3 as suggested by Yentes et al. (2013)], and:

$$
C_{i m}(r)=\frac{n_{i m}}{N-m+1}
$$

Where $C_{i m}$ is the fraction of patterns of length, $n_{i m}$ is the number of patterns that are similar between two sets (given the similarity criterion, $r$ ), $N$ is the data length, and $m$ is the embedding dimension. 


\section{Fractal Dimension}

The Fractal dimension (D) as an index of complexity of the stroke cycles was computed by the Higuchi's algorithm (Higuchi, 1988):

$$
D=\frac{d \log N(L(k))}{d \log (k)}
$$

Where $D$ is the fractal dimension and $N$ is the number of points from the speedtime series, $k$ is the integer and $L$ the length of the time series. The box-counting and the Higuchi's algorithm are the methods most often reported to quantify the fractal dimension (Wang, Sourina \& Nguyen, 2011). The box-counting is a reliable method assessing geometric forms (Liu, Zhang \& Yue, 2003). The Higuchi's algorithm is suitable for time series data as in the case of our research (Castiglioni, di Rienzo, Parati \& Faini, 2011).

\section{Statistical Procedures}

Data normality was tested by the Kolmogorov-Smirnov test, described as mean \pm $1 S D$ and $95 \%$ of the confidence interval.

Two-way repeated-measures ANOVAs (between $\times$ within-subject analysis; group $\times$ swim stroke; 3 levels of expertise $\times 4$ swim strokes; $p \leq .05$ ) was performed for each selected variable. Whenever needed, analysis of the variations and differences between conditions was carried out by one-way or repeated-measures ANOVAs plus Bonferroni post hoc test $(p \leq .05)$. All assumptions to run ANOVAs have been checked beforehand (data are normally distributed, independent samples, equal variances, same sample size) and sphericity tested.

Effect size was computed by the eta-squared $\left(\eta^{2}\right)$ and: without effect if $0<\eta^{2}$ $\leq 0.04$; minimum if $0.04<\eta^{2} \leq 0.25$; moderate if $0.25<\eta^{2} \leq 0.64$ and; strong if $\eta^{2}$ $>0.64$. Whenever suitable and appropriate, Cohen's d was also computed for the post hoc testing showing: (i) small effect size if $0 \leq \mathrm{dl} \leq 0.2$; (ii) moderate effect size if $0.2<|\mathrm{d}| \leq 0.5$ and; (iii) large effect size if $|\mathrm{d}|>0.5$.

\section{Results}

Significant and strong main effects of the expertise level $\left(\mathrm{F}_{2,72}=208.84 ; p<.001\right.$; $\left.\eta^{2}=0.75\right)$ and swim stroke $\left(\mathrm{F}_{3,72}=69.511 ; p<.001 ; \eta^{2}=0.66\right)$ were found in the swim speed. There was an expertise $\mathrm{x}$ swim stroke interaction $\left(\mathrm{F}_{6,72}=3.564\right.$; $\left.p<.001 ; \eta^{2}=0.13\right)$ in the swim speed. Performance increased with the level of expertise $(p<.001)$. Front-crawl was the fastest stroke, followed by the Butterfly, Backstroke and Breaststroke being the slowest $(p<.001)$.

\section{Speed Fluctuation}

Moderate-strong main effects of the expertise level $\left(p<.001 ; \eta^{2}=0.20\right)$ and the swim stroke $\left(p<.001 ; \eta^{2}=0.84\right)$ were verified (Table 1$)$. No Expertise $\times$ Stroke interaction was found $\left(p=.07 ; \eta^{2}=0.01\right)$. 
Follow-up analysis showed that compared with the highly qualified experts ( $p$ $<.001 ; 0.26 \leq \mathrm{d} \leq 1.24)$ and expert swimmers $(p<.001 ; 0.26 \leq \mathrm{d} \leq 1.22)$, nonexpert swimmers had a higher dv with a large effect size in three of the swim strokes (Front-crawl, Backstroke and Butterfly). No differences were found between the two top-tier groups (highly qualified experts vs. experts). The absence of differences between highly qualified expert and expert swimmers can also be verified by the overlapping of the $95 \%$ confidence interval limits of both groups. There was no significant difference between Front-crawl and Backstroke. Breaststroke showed a higher $\mathrm{dv}$ than the remaining strokes $(p<.001)$. Overall, the $95 \%$ confidence interval ranges roughly between 36 and $43 \%$. Butterfly stroke is more prone to a higher dv than Front-crawl and Backstroke $(p<.001)$. Its $95 \%$ confidence interval ranges between 23 and 35\%, while it ranges between 11 and 22\% for Front-crawl and Backstroke.

Altogether, breaststroke featured the highest dv, followed by the Butterfly, then Front-crawl and Backstroke with similar magnitudes in all groups of expertise. The $\mathrm{dv}$ was higher in nonexpert swimmers than expert or highly qualified expert swimmers.

\section{Approximate entropy}

A moderate main effect of the swim stroke $\left(p<.001 ; \eta^{2}=0.41\right)$ and a group trivial effect $\left(p=.12 ; \eta^{2}=0.05\right)$ was found for the ApEn (Table 2). There was an Expertise $\times$ Stroke interaction $\left(p<.001 ; \eta^{2}=0.06\right)$.

\section{Table 1 The Variation of the Speed Fluctuation (dv) Across the Three Different Levels of Expertise}

\begin{tabular}{|c|c|c|c|c|}
\hline & \multicolumn{4}{|c|}{ Speed Fluctuation (dv, \%) } \\
\hline & Front-crawl & Backstroke & Breaststroke & Butterfly \\
\hline Highly qualified & $\begin{array}{c}15.11 \pm 2.97 \\
(13.88-16.33)\end{array}$ & $\begin{array}{c}14.31 \pm 6.35 \\
(11.68-16.92)\end{array}$ & $\begin{array}{c}39.44 \pm 6.66 \\
(36.68-42.18)\end{array}$ & $\begin{array}{c}25.51 \pm 3.80 \\
(23.94-27.07)\end{array}$ \\
\hline Experts & $\begin{array}{c}15.57 \pm 2.91 \\
(14.27-16.86)\end{array}$ & $\begin{array}{c}14.11 \pm 4.12 \\
(12.43-15.77)\end{array}$ & $\begin{array}{c}39.72 \pm 4.13 \\
(38.00-41.43)\end{array}$ & $\begin{array}{c}25.17 \pm 4.74 \\
(23.21-27.12)\end{array}$ \\
\hline \multirow[t]{3}{*}{ Nonexperts } & $\begin{array}{c}18.40 \pm 6.00 \\
(15.41-21.38)\end{array}$ & $\begin{array}{c}18.94 \pm 8.75 \\
(15.41-22.45)\end{array}$ & $\begin{array}{c}41.19 \pm 6.69 \\
(38.48-43.88)\end{array}$ & $\begin{array}{c}32.44 \pm 6.92 \\
(29.06-35.81)\end{array}$ \\
\hline & \multicolumn{4}{|c|}{ ANOVA } \\
\hline & DoF & $\mathbf{F}$ & $p$ & $\eta^{2}$ \\
\hline $\begin{array}{l}\text { Expertise } \times \\
\text { stroke interaction }\end{array}$ & 6,72 & 1.935 & .07 & .01 \\
\hline $\begin{array}{l}\text { Expertise level } \\
\text { effect }\end{array}$ & 2,72 & 9.017 & $<.001$ & .20 \\
\hline $\begin{array}{l}\text { Swim stroke } \\
\text { effect }\end{array}$ & 3,72 & 404.611 & $<.001$ & .84 \\
\hline
\end{tabular}

Note. Reported in mean \pm 1 standard-deviation $(S D)$ and $95 \%$ of confidence interval $(95 \mathrm{CI})$. 
There were significant differences among all pairwise comparison $(p<.001)$, except between Front-crawl and Butterfly $(p=.75)$. Backstroke $(p<.001)$ showed the highest ApEn, while Breaststroke the lowest $(p<.001)$ in comparison with the remaining swim strokes. Because there was an Expertise $\times$ Stroke interaction, univariate analysis was carried out to further investigate the hypothetical effect of the group. There were no significant variations at Front-Crawl $\left(\mathrm{F}_{2,72}=1.668 ; p=\right.$ $\left..19 ; \eta^{2}=0.05\right)$ and Butterfly $\left(\mathrm{F}_{2,72}=1.565 ; p=.21 ; \eta^{2}=0.05\right)$ but significance was found for Backstroke $\left(\mathrm{F}_{2,72}=3.412 ; p=.04 ; \eta^{2}=0.05\right)$ and Breaststroke $\left(\mathrm{F}_{2,72}=5.251\right.$; $\left.p=.01 ; \eta^{2}=0.13\right)$. Pairwise comparison revealed moderate-large effect sizes at Front-crawl $(0.26 \leq \mathrm{d} \leq 0.58)$, Backstroke $(0.32 \leq \mathrm{d} \leq 0.84)$ and Breaststroke $(0.20$ $\leq \mathrm{d} \leq 0.88)$ but small-moderate effect sizes at Butterfly $(0.00 \leq \mathrm{d} \leq 0.50)$.

In this sense, a higher predictability of the intercyclic variations is found at Breaststroke and a random one at Backstroke. Regarding the expertise level, mixed findings were obtained. While ApEn shows a slight trend to be more predictable at Front-Crawl and Butterfly stroke in top-tier swimmers, the opposite might be happening at the two other swim strokes.

\section{Fractal dimension}

There was a moderate effect of the swim stroke $\left(p<.001 ; \eta^{2}=0.41\right)$ and a small effect of the expertise level $\left(p=.01 ; \eta^{2}=0.12\right.$; Table 3$)$. However, we failed to find an Expertise $\times$ Stroke interaction $\left(p=.13 ; \eta^{2}=0.03\right)$

\section{Table 2 The Variation of the Approximate Entropy (ApEn) Across the Three Different Levels of Expertise}

\begin{tabular}{|c|c|c|c|c|}
\hline & \multicolumn{4}{|c|}{ Approximate Entropy (ApEn, dimensionless) } \\
\hline & Front-crawl & Backstroke & Breaststroke & Butterfly \\
\hline Highly qualified & $\begin{array}{l}0.66 \pm 0.12 \\
(0.60-0.71)\end{array}$ & $\begin{array}{l}0.83 \pm 0.15 \\
(0.76-0.89)\end{array}$ & $\begin{array}{l}0.65 \pm 0.11 \\
(0.60-0.69)\end{array}$ & $\begin{array}{l}0.73 \pm 0.10 \\
(0.68-0.77)\end{array}$ \\
\hline Experts & $\begin{array}{l}0.69 \pm 0.11 \\
(0.64-0.73)\end{array}$ & $\begin{array}{l}0.87 \pm 0.09 \\
(0.82-0.91)\end{array}$ & $\begin{array}{l}0.67 \pm 0.08 \\
(0.63-0.70)\end{array}$ & $\begin{array}{l}0.73 \pm 0.11 \\
(0.68-0.77)\end{array}$ \\
\hline \multirow[t]{3}{*}{ Nonexperts } & $\begin{array}{l}0.73 \pm 0.12 \\
(0.67-0.77)\end{array}$ & $\begin{array}{l}0.77 \pm 0.14 \\
(0.71-0.83)\end{array}$ & $\begin{array}{l}0.58 \pm 0.12 \\
(0.52-0.62)\end{array}$ & $\begin{array}{l}0.68 \pm 0.10 \\
(0.64-0.72)\end{array}$ \\
\hline & \multicolumn{4}{|c|}{ ANOVA } \\
\hline & DoF & $\mathbf{F}$ & $p$ & $\eta^{2}$ \\
\hline $\begin{array}{l}\text { Expertise } \times \\
\text { stroke interaction }\end{array}$ & 6,72 & 3.880 & $<.001$ & 0.06 \\
\hline $\begin{array}{l}\text { Expertise level } \\
\text { effect }\end{array}$ & 2,72 & 2.106 & .12 & 0.05 \\
\hline $\begin{array}{l}\text { Swim stroke } \\
\text { effect }\end{array}$ & 3,72 & 55.175 & $<.001$ & 0.41 \\
\hline
\end{tabular}

Note. Reported in mean \pm 1 standard-deviation $(S D)$ and $95 \%$ of confidence interval $(95 \mathrm{CI})$. 
Table 3 The Variation of the Fractal Dimension (D) Across the Three Different Levels of Expertise

\begin{tabular}{|c|c|c|c|c|}
\hline & \multicolumn{4}{|c|}{ Fractal Dimension (D, dimensionless) } \\
\hline & Front-crawl & Backstroke & Breaststroke & Butterfly \\
\hline Highly qualified & $\begin{array}{l}1.84 \pm 0.08 \\
(1.80-1.87)\end{array}$ & $\begin{array}{l}1.83 \pm 0.06 \\
(1.79-1.85)\end{array}$ & $\begin{array}{l}1.92 \pm 0.02 \\
(1.90-1.93)\end{array}$ & $\begin{array}{l}1.88 \pm 0.07 \\
(1.85-1.91)\end{array}$ \\
\hline Experts & $\begin{array}{l}1.85 \pm 0.09 \\
(1.81-1.88)\end{array}$ & $\begin{array}{l}1.85 \pm 0.06 \\
(1.82-1.87)\end{array}$ & $\begin{array}{l}1.92 \pm 0.03 \\
(1.90-1.93)\end{array}$ & $\begin{array}{l}1.88 \pm 0.06 \\
(1.84-1.90)\end{array}$ \\
\hline \multirow[t]{3}{*}{ Nonexperts } & $\begin{array}{l}1.89 \pm 0.06 \\
(1.86-1.91)\end{array}$ & $\begin{array}{l}1.88 \pm 0.04 \\
(1.86-1.90)\end{array}$ & $\begin{array}{l}1.94 \pm 0.02 \\
(1.92-1.95)\end{array}$ & $\begin{array}{l}1.92 \pm 0.04 \\
(1.90-1.94)\end{array}$ \\
\hline & \multicolumn{4}{|c|}{ ANOVA } \\
\hline & DoF & $\mathbf{F}$ & $\mathbf{P}$ & $\eta^{2}$ \\
\hline $\begin{array}{l}\text { Expertise } \times \\
\text { stroke interaction }\end{array}$ & 6,72 & 1.661 & 0.13 & 0.03 \\
\hline $\begin{array}{l}\text { Expertise level } \\
\text { effect }\end{array}$ & 2,72 & 5.070 & 0.01 & 0.12 \\
\hline $\begin{array}{l}\text { Swim stroke } \\
\text { effect }\end{array}$ & 3,72 & 51.689 & $<0.001$ & 0.41 \\
\hline
\end{tabular}

Note. Reported in mean \pm 1 standard-deviation $(S D)$ and $95 \%$ of confidence interval (95CI).

The fractal dimension was higher with large effect sizes in the group of nonexperts than the other two (highly qualified experts: $p=.01,0.70 \leq \mathrm{d} \leq 1.0$; experts: $p$ $=.05,0.52 \leq \mathrm{d} \leq 0.78)$. The variable was different among all pair wises $(p<.001)$ except in Front-crawl vs. Backstroke. Hence, Breaststroke presented the highest level of complexity followed by Butterfly, Front-crawl and Backstroke. This level of intracyclic complexity is clearly higher for nonexpert swimmers than their better counterparts. In the four strokes we can see a shift of the $95 \%$ confidence interval band to the left side (i.e., a decrease of the fractal dimension) comparing nonexperts with competitive swimmers. For instance, in Front-crawl the confidence interval is $1.86-1.91$ for nonexperts and about $1.80-1.88$ for highly qualified experts and experts.

\section{Discussion}

The aim was to examine the variation of linear and nonlinear behavior in subjects with different levels of swimming expertise among the four swim strokes. Swimming does exhibit nonlinear properties but its magnitude differs according to the swim stroke and level of expertise of the performer.

For a long time, motor control was concealed under a cognitivist perspective inspired by Cybernetics, such as the schema theory by Schmidt. The motor program is made up of an invariant part, the plan and parameters that allow the plan 
to be fulfilled under a given setting (Komar, Seifert \& Thouvarecq, 2015). Hence, a motor behavior that drifts away from the plan is considered as the result of an error or mistake and associated to low levels of expertise or poor performances. Eventually this framework has been challenged by the complex systems approach (Davids et al., 2014). This approach encompasses the main features and assumptions of the nonlinear dynamical systems. We can find a set of features that interact among themselves in a dynamical fashion. This can be deterministic (i.e., at a given moment one single future state of the system can happen, depending on the present state) or stochastic (i.e., a random later state). As shared earlier, it is nonlinear because there is no direct proportionality between input and output in the system. Applying these concepts to motor behavior, the latter depends upon several factors and the interrelationship among them, making it a highly complex system. In such circumstances, different motor behaviors (i.e., behavior variability) to reach a given performance outcome are no longer considered as errors or mistakes but a proxy of adaptability (i.e., a ratio between stability regardless of external changes and flexibility accommodating to a dynamic environment) (Komar, Seifert \& Thouvarecq, 2015). On top of that, small changes in the motor behavior that are undetected by mainstream and classical measures may have a large effect in the output. This could explain why it is often challenging to have a deep understanding of some phenomena regarding motor behavior. Thus, nonlinear parameters can be quite useful and enlightening. Because of its highly complex nature and lack of full understanding, some research groups have addressed the concern that motor behavior complexity should be a main topic of interest in swimming (Barbosa, Silva, Reis, Costa, Garrido, Policarpo \& Reis, 2010b; Seifert, Komar, et al., 2014a).

There was no Expertise $\times$ Stroke interaction for the $d v$, while moderate-strong main effects of the expertise level and swim stroke were verified. Breaststroke featured the highest dv, followed by the Butterfly, then Front-crawl and Backstroke with similar magnitudes. This same order was reported earlier in the literature and is due to a set of biomechanical (i.e., environmental) constraints that are imposed on the swimmer performing different strokes (Craig \& Pendergast, 1979; Barbosa et al., 2013). The dv is the balance between two main external forces acting upon the swimmer: the thrust and the drag. If the thrust increases for a given drag, likewise, the speed increases; whereas for a given thrust, in the event of an increasing drag, the speed decreases. Hence, swim strokes producing more thrust and drag impose a higher $\mathrm{dv}$. It is also reported that there is a relationship between the $\mathrm{dv}$ and energy expenditure. The energy expenditure would be minimized if the swim motion was uniform (i.e., $\mathrm{dv}=0 \%$ ). In fact each time there is a change in the speed within a stroke cycle, the body needs to overcome inertia, resulting in higher energy expenditure (Barbosa, Bragada, Reis, Marinho, Carvalho \& Silva, 2010a). The $\mathrm{dv}$ was higher in nonexpert swimmers than their expert or highly qualified expert counterparts. It has also been reported as a trend for $\mathrm{dv}$ to decrease with increasing speeds or swim paces (Manley \& Atha, 1992; Barbosa et al., 2013; Cohen et al., 2014). Therefore as far as one is concerned, the $d v$ is a linear parameter that is sensitive to the expertise level in swimming. That said, the selection of multiple nonlinear measures including linear parameters enhances the behavioral discriminations, allowing a better understanding of different motor behaviors, and should not be disregarded (Neumeister et al., 2004). 
The ApEn was selected to monitor the intercyclic variations over the trials; where the lower the value, the more predictable the time-series is. An Expertise $x$ Swim stroke interaction was reported where a lower ApEn was found at Breaststroke than at Backstroke. This parameter was reported as being determined by sensory inputs, motor control mechanisms and biomechanical behavior (Arif et al., 2004). The findings reported earlier can be related to the interlimb synchronization and the body positioning requirements of different swim strokes. Breaststroke involves the simultaneous action of both arms and legs. The arms feature only 3 phases (outsweep, insweep and recovery) and the legs feature 4 phases (outsweep, downsweep, insweep and recovery) making it the most predictable of the four techniques. On the other hand, backstroke features a supine position (changing several sensory inputs by vision and vestibular systems) concurrent to the synchronization of four alternated limbs (the arms must each perform 4-5 phases and the legs 6 kicks) almost in antiphase, plus the body rotation (in the longitudinal axis to each side). These challenging features can explain the higher intercyclic randomness of this stroke. Top-tier swimmers showed a low ApEn at Front-crawl and Butterfly stroke. This is reflective of a previous study where young swimmers that were monitored over a season reported an improvement in their performance in relation to a decrease in ApEn (Barbosa et al., 2015). The shifts in the balance between thrust and drag over consecutive cycles changes not only the body's acceleration and speed but also the energy cost of swimming (Barbosa et al., 2010a). Therefore, expert swimmers are aware that keeping an "even" and similar speed pattern over the trial will minimize the energy cost and improve the swimming efficiency. As far as land-based gait goes, ApEn is higher for elderly than young subjects at selected paces (Arif, Ohtaki, Nagatomi \& Inooka, 2004). Hence, it seems that with higher levels of expertise the ApEn is prone to diminish, regardless of land-based or in-water locomotion.

The D provides insight on the complexity and irregularity of intracyclic variations. The higher the parameter, the more complex and irregular the time-series is. There was no Expertise $\times$ Stroke interaction. Nevertheless, main effects of the swim stroke and the expertise level were obtained. Breaststroke showed the highest D followed by Butterfly, Front-crawl and Backstroke in all groups of expertise level. The D was higher in nonexpert swimmers than their better counterparts, again in all groups of expertise level. The level of complexity can be due to different constraints acting upon the swimmer. Hence, the constraint-led approach can provide a framework explaining it (Davids et al., 2014). The D may be the result of the interaction between individual, environmental and task constraints. As shared earlier, the propulsive and resistive forces produced or acting on the swimmer are different among the four strokes (environmental constraints). To reach a given speed or pace, different combinations of stroke rate and stroke length can be selected by the subject (task constraints). At least for the top-tier swimmers, their specialty (best stroke or distance, i.e., swim event) can also play a role (organismic constraint). The higher level of irregularity for nonexpert swimmers than their top-tier counterparts seem to be aligned with the understanding that significant changes in the speed within a stroke cycle will lead to a higher energy cost and lower swim efficiency (Barbosa et al., 2010a). In on-land locomotion, this parameter was significantly higher in elderly than young participants (Sekine, Tamura, Akay, Fujimoto, Togawa \& Fukui, 2002). Our findings in swimming seem to be in tandem with these findings. Alto- 
gether, this suggests that a lower level of complexity may be found in high levels of expertise in both on-land and in-water locomotion.

Overall, the effects seem to be large when comparing nonexpert swimmers with both the top-tier groups, despite small-moderate effects between the latter ones. Nonexpert swimmers exhibited a higher level of complexity than expert and highly qualified expert swimmers. This supports a similar finding that compared the elbow-knee continuous relative phase at Breaststroke between recreational and competitive swimmers (Seifert, Leblanc, Hérault, Komar, Button \& Chollet, 2011). To become an expert swimmer, subjects must undergo a considerable amount of practice and instruction, and at this stage of learning, interindividual coordination variability is compressed to a narrower range of movement solutions. A research question under this topic is to explore the types of effective deliberate practice based on linear or a nonlinear pedagogy (Chow, Davids, Button, Shuttleworth, Renshaw \& Araújo, 2007). It has been suggested that to achieve world-class standards, elite swimmers must once again explore the environment to optimize their technique to leverage over direct contenders (Seifert et al., 2011). Unfortunately we failed to obtain evidence on this because for most of the parameters selected, there were no significant differences and/or small effect sizes between expert and highly qualified expert swimmers. As periodization was not considered in this study, it forms a possible limitation. While data collection was held at the same time in both groups, there was a time lag between the main competitions of the season for them. While the expert swimmers were tapering for the main competition of their season and in their peak performance, the highly qualified experts were still building up because the most important competition was still 6-8 weeks away. As the gap between experts and highly qualified experts are theoretically narrow, this explanation could point out why meaningful differences were not found between these two groups (Chow, Davids, Button, Shuttleworth, Renshaw \& Araújo, 2007).

Our main finding is that swimming is indeed a highly complex locomotion technique, even more so for subjects with lower levels of expertise. E.g., walking on-land, the fractal dimension was reported as ranging between 1.10 and 1.40 (Schiffman, Chelidze, Segala, \& Hasselquist, 2009). Our highly qualified expert swimmers (the ones with lower complexity scores) showed a fractal dimension range of 1.80-1.90. Fractal dimension it is expected to range between 0 and 3. Higher fractal dimension, likewise a more complexity of the behavior is being shown. The explanation for this higher complexity of in-water locomotion may rely on three reasons. In an aquatic environment there are four main external forces acting on the body (buoyancy, weight, thrust and drag); whereas on land the number of forces is reduced to just the weight and thrust as buoyancy and drag forces can be neglected. As expected, a higher number of forces impose a higher complexity in the control of the system. This may be valid for swimming, and also for other water sports such as canoeing, rowing, etc. Although evidence on the latter ones has yet to be found. Another explanation for the higher complexity relies on the meaningful changes that do happen in several sensory systems when in water. Having the face immersed, the visual information will be affected. In addition, swimming is characterized by staying in the horizontal position, which impacts the vestibular information. Last but not least, the input in the somatosensory system in water is also quite different from what is experienced on land. The third reason is the different number of seg- 
ments to be synchronized over a cycle (i.e., interlimb coordination). On land there are two main propulsive segments (lower limbs) and two other segments (upper limbs) helping to keep the balance. In water, at least four propulsive limbs are used (both upper and lower limbs) and in some circumstances, such as Butterfly stroke, the wave motion by the torso can add some extra thrust.

Future research should consider the periodization plan, such that the hypothetical relationships between nonlinear parameters, hydrodynamics, kinematics, interlimb coordination and neuromuscular response can be further understood. Eventually, research may provide some understanding if and how nonlinear pedagogy is more effective than other approaches in helping one to excel and improve his expertise level in swimming.

\section{Conclusions}

As a conclusion, swimming does exhibit nonlinear properties and encompasses a "typical" complex system. The magnitudes of the linear and nonlinear parameters are indeed different according to the swim stroke and level of expertise of the performer. The effects seemed to be quite large for the nonexpert swimmers in comparison with both top-tier groups; while between the latter ones, the effects were overall small-moderate.

\section{Acknowledgments}

The authors would like to express their deep gratitude to Mr. Lim Aik Ho, Mr. Huang Wei Lun and Ms. Chen Simin for their help with the data collection. This research was funded by the NIE AcRF grant (RI 11/13 TB).

\section{References}

Abarbanel, H.D., Rabinovich, M.I., \& Sushchik, M.M. (1993). Introduction to nonlinear dynamics for physicists (Vol. 53, pp. 158). Singapore: World Scientific. doi:10.1142/2036

Arif, M., Ohtaki, Y., Nagatomi, R., \& Inooka, H. (2004). Estimation of the effect of cadence on gait stability in young and elderly people using approximate entropy technique. Measurement Science Review, 4, 29-40.

Barbosa, T.M., Bragada, J.A., Reis, V.M., Marinho, D.A., Carvalho, C., \& Silva, A.J. (2010a). Energetics and biomechanics as determining factors of swimming performance: updating the state of the art. Journal of Sports, Science, and Medicine, 13, 262-269. PubMed doi:10.1016/j.jsams.2009.01.003

Barbosa, T., Silva, A.J., Reis, A.M., Costa, M., Garrido, N., Policarpo, F., \& Reis, V.M. (2010b). Kinematical changes in swimming front Crawl and Breaststroke with the AquaTrainer snorkel. European Journal of Applied Physiology, 109(6), 1155-1162. PubMed doi:10.1007/s00421-010-1459-x

Barbosa, T.M., Morouço, P.G., Jesus, S., Feitosa, W.G., Costa, M.J., Marinho, D.A., . . . Garrido, N.D. (2013). The interaction between intra-cyclic variation of the velocity and mean swimming velocity in young competitive swimmers. International Journal of Sports Medicine, 34(2), 123-130. PubMed 
Barbosa, T.M., Morais, J.E., Marques, M.C., Silva, A.J., Marinho, D.A., \& Kee, Y.H. (2015). Hydrodynamic profile of young swimmers: changes over a competitive season. Scandinavian Journal of Medicine \& Science in Sports, 25, 184-196. PubMed doi:10.1111/ sms. 12281

Bravi, A., Longtin, A., \& Seely, A.J. (2011). Review and classification of variability analysis techniques with clinical applications. Biomedical Engineering Online, 10, 90. PubMed doi:10.1186/1475-925X-10-90

Castiglioni, P., Di Rienzo, M., Parati, G., \& Faini, A. (2011). Fractal dimension of mean arterial pressure and heart-rate time series from ambulatory blood pressure monitoring devices. IEEE. Computers in Cardiology, September, 593-596.

Chow, J.Y., Davids, K., Button, C., Shuttleworth, R., Renshaw, I., \& Araújo, D. (2007). The role of nonlinear pedagogy in physical education. Review of Educational Research, 77(3), 251-278. doi:10.3102/003465430305615

Cohen, R.C., Cleary, P.W., Harrison, S.M., Mason, B.R., \& Pease, D.L. (2014). Pitching effects of buoyancy during four competitive swimming strokes. Journal of Applied Biomechanics, 30(5), 609-618. PubMed doi:10.1123/jab.2013-0260

Craig, A.B., \& Pendergast, D.R. (1979). Relationships of stroke rate, distance per stroke, and velocity in competitive swimming. Medicine and Science in Sports and Exercise, 11(3), 278-283. PubMed doi:10.1249/00005768-197901130-00011

Craig, A.B., Termin, B., \& Pendergast, D.R. (2006). Simultaneous recordings of velocity and video during swimming. Portuguese Journal of Sport Sciences, 6(2), 32-35.

Davids, K., Hristovski, R., Araùjo, D., Balague Serre, N., Button, C., \& Passos, P. (2014). Complex Systems in Sport. New York, NY: Routledge.

Figueiredo, P., Kjendlie, P.L., Vilas-Boas, J.P., \& Fernandes, R.J. (2012). Intracycle Velocity Variation of the Body Centre of Mass in Front Crawl? International Journal of Sports Medicine, 33, 285-290. PubMed doi:10.1055/s-0031-1301323

Higuchi, T. (1988). Approach to an irregular time series on the basis of the fractal theory. Physica, 31, 277-283.

Komar, J., Seifert, L., \& Thouvarecq, R. (2015). What Variability tells us about motor expertise: measurements and perspectives from a complex system approach. Movement \& Sport Sciences-Science \& Motricité, (89), 65-77

Liu, J.Z., Zhang, L.D., \& Yue, G.H. (2003). Fractal dimension in human cerebellum measured by magnetic resonance imaging. Biophysical Journal, 85, 4041-4046. PubMed doi:10.1016/S0006-3495(03)74817-6

Manley, P.K., \& Atha, J. (1992). Intra-stroke velocity fluctuations in paced breaststroke swimming. Biomechanics and medicine in swimming VI (pp. 151-160). London: E $\&$ FN Spon.

Neumeister, H., Cellucci, C.J., Rapp, P.E., Korn, H., \& Faber, D.S. (2004). Dynamical analysis reveals individuality of locomotion in goldfish. The Journal of Experimental Biology, 207, 697-708. PubMed doi:10.1242/jeb.00811

Pincus, S.M. (1991). Approximate entropy as a measure of system complexity. Proceedings of the National Academy of Sciences of the United States of America, 88, 2297-2301. PubMed doi:10.1073/pnas.88.6.2297

Psycharakis, S.G., \& Sanders, R.H. (2009). Validity of the use of a fixed point for intracycle velocity calculations in swimming. Journal of Science and Medicine in Sport, 12, 262-265. PubMed doi:10.1016/j.jsams.2007.11.008

Schiffman, J.M., Chelidze, D., Segala, D.B., \& Hasselquist, L. (2009). Nonlinear analysis of gait kinematics to track changes in oxygen consumption in prolonged load carriage walking: a pilot study. Journal of Biomechanics, 18, 2196-2199. PubMed doi:10.1016/j. jbiomech.2009.06.011 
Seifert, L., Leblanc, H., Hérault, R., Komar, J., Button, C., \& Chollet, D. (2011). Inter-individual variability in the upper-lower limb breaststroke coordination. Human Movement Science, 30(3), 550-565. PubMed doi:10.1016/j.humov.2010.12.003

Seifert, L., Komar, J., Crettenand, F., \& Millet, G. (2014a). Coordination Pattern Adaptability: Energy Cost of Degenerate Behaviors. PLoS One, 25;9(9), e107839.

Seifert, L., Komar, J., Barbosa, T., Toussaint, H., Millet, G., \& Davids, K. (2014b). Coordination pattern variability provides functional adaptations to constraints in swimming performance. Sports Medicine (Auckland, N.Z.), 44(10), 1333-1345. PubMed doi:10.1007/s40279-014-0210-x

Sekine, M., Tamura, T., Akay, M., Fujimoto, T., Togawa, T., \& Fukui, Y. (2002). Discrimination of walking patterns using wavelet-based fractal analysis. Neural Systems and Rehabilitation Engineering. IEEE Transactions on, 10, 188-196.

Wang, Q., Sourina, O., \& Nguyen, M.K. (2011). Fractal dimension based neurofeedback in serious games. The Visual Computer, 27, 299-309. doi:10.1007/s00371-011-0551-5

Yentes, J.M., Hunt, N., Schmid, K.K., Kaipust, J.P., McGrath, D., \& Stergiou, N. (2013). The Appropriate use of approximate entropy and sample entropy with short data sets. Annals of Biomedical Engineering, 41, 349-365. PubMed doi:10.1007/s10439-012-0668-3 
Copyright of Motor Control is the property of Human Kinetics Publishers, Inc. and its content may not be copied or emailed to multiple sites or posted to a listserv without the copyright

holder's express written permission. However, users may print, download, or email articles for individual use. 